\title{
The relevance of plasma R-spondin 1 and Slit2 as predictive biomarkers in cervical cancer chemotherapy and radiotherapy
}

\author{
Jichan Nie, Jun Shao, Sun-Wei Guo, Xishi Liu \\ Gynecology Department, Shanghai Obstetrics and Gynecology Hospital of Fudan University, Shanghai, China \\ Contributions: (I) Conception and design: J Nie, X Liu; (II) Administrative support: S Guo; (III) Provision of study materials or patients: J Nie, J Shao; \\ (IV) Collection and assembly of data: All authors; (V) Data analysis and interpretation: J Nie, X Liu; (VI) Manuscript writing: All authors; (VII) Final \\ approval of manuscript: All authors. \\ Correspondence to: Xishi Liu; Sun-Wei Guo. Shanghai Obstetrics and Gynecology Hospital of Fudan University, 419 Fangxie Road, Shanghai 200011, \\ China.Email: liu_xishi@fudan.edu.cn; hoxa10@outlook.com.
}

\begin{abstract}
Background: R-spondin 1 (Rspol) and Slit2 have been found to play a vital role in cancer development, and have the potential to act as therapeutic adjuvants to increase tolerance to aggressive chemotherapy and/ or radiotherapy. This "proof of concept" study evaluates the role of Rspo1 and Slit2 expression in the clinical outcome of cervical cancer patients.

Methods: Using enzyme linked immunosorbent assays (ELISA), we analyzed Rspo1 and Slit2 levels from patients diagnosed with the International Federation of Gynecology and Obstetrics (FIGO) stage IB1-IIA2 cervical cancer ( $\mathrm{n}=34)$ who received chemotherapy $(\mathrm{CT})$ and/or radiotherapy $(\mathrm{RT})$ and correlated the data with the acute radiation morbidity scoring criteria.

Results: Cervical cancer patients who underwent CT and/or RT showed that neither the level of Rspo1 nor the level of Slit2 changed significantly after the first round of CT (CT1), RT, or the second CT (CT2). However, neurological sensory scores and influence of infection scores were elevated following increasing rounds of therapies. Rspo1 levels correlated negatively with the morbidity score of neutrophils, hemoglobin, platelet, infection score, neurological sensory score, and performance status after CT1, RT, or CT2. We also found that Slit2 levels were negatively correlated with genitourinary, heart, and neurological sensory scores at RT and CT2.

Conclusions: The levels of Rspo1 and Slit2 correlate positively to the tolerance of the patients. In contrast, the levels of Rspo1 and Slit2 showed a negative correlation to the morbidity score of the patients undergoing CT and/or RT. Thus, Rspo1 and Slit2 may be potential predictive biomarkers for patients with cervical cancer receiving CT or RT postoperatively, which supports the current pursuit of the clinical significance of Rspo1 and Slit2.
\end{abstract}

Keywords: R-spondin 1; Slit2; cervical cancer; chemotherapy; radiotherapy

Submitted Dec 10, 2020. Accepted for publication Apr 29, 2021.

doi: $10.21037 / \mathrm{atm}-21-87$

View this article at: http://dx.doi.org/10.21037/atm-21-87

\section{Introduction}

Despite significant advances in the development of screening methods and treatment technologies, cervical cancer therapy remains far from satisfactory and, of the major malignancies, cervical cancer remains the fourth leading cause of death for women worldwide, including those with a known family history $(1,2)$. Nearly all cervical cancers are caused by the human papilloma virus (HPV) and just two HPV subtypes, 16 and 18, are responsible for approximately $70 \%$ of all cases (3). For more advanced or recurrent cervical cancer, multimodal therapy shows potential benefits with 
radiation therapy in combination with chemotherapy (2). Furthermore, in cases of aggressive recurrence of cervical cancer, a radical surgical approach is often adopted where the radiation treatment can be delivered either prior to surgery, concurrently, or postoperatively based on the prescribed treatment plan (4). Although improved disease control and survival have been achieved by radiotherapy in combination with chemotherapy, the treatment outcomes from external beam RT (EBRT) and brachytherapy (BRT) remain poor, and patients in the advanced stage of cervical cancer are at a high risk of recurrence or death, insofar as the 5 -year overall survival rate is only $67 \%$ and nearly half $(44 \%)$ of patients will experience a relapse $(4,5)$. Furthermore, treatment efficacy and prognosis varies according to the different radiosensitivity of individual patients, with findings that some gene products improved the sensitivity of tumor cells via cell cycle control, apoptosis regulation, and DNA damage (6). Thus, precisely predicting treatment outcomes as a result of cervical cancer therapy remains problematic. One possible approach is to investigate the specific biomarkers in cervical cancer and advanced molecular profiling of the tumor, eventually predict the individual patient's prognosis and response to radiotherapy more accurately (7).

Slit2, a human homolog of the Drosophila Slit2 gene, is one of the most important members of the SLIT family of large secreted proteins, and has been shown to guide axons and induce neuron migration by interacting with Robo receptors (8-10). Besides its vital role in guiding the projection of normal axons and neuron development, Slit2 is also expressed in other non-neuronal tissues such as the heart, lungs, and kidneys, indicating other significant roles in addition to axon guidance (11). Furthermore, it has been recently confirmed that Slit actively participates in tumor development. For instance, Wang et al. demonstrated that Slit secreted by cancer cells could attract vascular endothelia cells for the development of tumor-associated blood vessels to further support tumor growth, as evidenced by angiogenesis in a xenograft model of human melanoma cells (11). In addition, Slit-Robo4 has also been shown to act as a chemoattractant that interacts with endothelia cells for angiogenesis (12). More recently, researchers found that higher tumor vasculogenesis was associated with an upregulation of Slit expression, as evidenced by an increased VEGF expression and microvessel density in a chemically induced squamous cell carcinoma model, suggesting that Slit could be an important biomarker that is closely associated with tumor development (13). To further validate this, a monoclonal antibody against Robo1, a Slit receptor, was applied to competitively block the interaction of SlitRobo. The results showed a significant inhibition of tumor angiogenesis after treatment with the antibody, suggesting the crucial role of Slit in tumor progression.

$\mathrm{R}$-spondin 1 (Rspo1) is an important member of a protein family containing secreted furin-like domains, which has been demonstrated to interact with the Wnt signaling pathway by stabilizing intracellular $\beta$-catenin and influencing externalization of the LRP6 receptor (14). Furthermore, the expression of Rspo1 has been found in mouse ovaries and other major organs $(15,16)$. In addition, Rspo1 has been found to play a critical role in ovarian cancer development and correlates positively with tumor progression $(17,18)$.

Due to the important roles of Rspo1 and Slit2 in tumor development, we hypothesized that clinical levels of Rspo1 and Slit 2 could be used to predict the treatment outcomes of cervical cancer in clinical settings, and their concentrations may thus be indicative of the tolerance to chemotherapy and/or radiotherapy. Therefore, we investigated the levels of Rspo1 and Slit2 as potential biomarkers in clinical patients receiving chemotherapy and/or radiotherapy postoperatively. We also examined the correlation between Rspo1 and Slit2 levels with postoperative chemotherapy or radiotherapy, and some putative prognostic variables from the acute radiation morbidity scoring criteria. We present the following article in accordance with the MDAR reporting checklist (available at http://dx.doi.org/10.21037/atm-21-87).

\section{Methods}

\section{Patients}

Between March 2015 and September 2015, a total of 34 confirmed cervical cancer patients aged 31-57 years (mean age 44.9 years) were admitted to the Obstetrics and Gynecology Hospital of Fudan University. All patients were staged clinically according to the International Federation of Gynecology and Obstetrics (FIGO) staging criteria, and patients with a diagnosis of stage IB1-IIA2 were included in the present study. The distribution of grades was as follows: stage IB1 $(n=21)$, stage IB2 $(n=4)$, stage IIA1 $(n=8)$, and stage IIA2 $(n=1)$. All procedures performed in this study involving human participants were in accordance with the Declaration of Helsinki (as revised in 2013). The study was approved by the ethics board of Shanghai Obstetrics and Gynecology Hospital of Fudan University (No.: 2021-27) 
and informed consent was taken from all the patients.

\section{Study design and treatments}

As phase I of the study, we recruited 34 patients with FIGO stage IB1 IIA2 who were scheduled to receive surgery in our hospital and who received chemotherapy and/or radiotherapy postoperatively. The recruited patients' age, gravidity, parity, height, weight, menopausal status (premenopausal or menopausal), pre-operative use of hormonal drugs (if any), pre-operative blood tests (including platelet counts, mean platelet volume, hematocrit, neutrophil count, aPTT, PT, TT, fibrinogen, INR, D-dimer (if tested), WBC, and Hb levels), and the presence or absence of chronic conditions, such as diabetes, hypertension, and cardiovascular disease were recorded. A $10 \mathrm{~mL}$ blood sample was collected in a heparin-containing tube and stored at $-20{ }^{\circ} \mathrm{C}$ for further use. The blood sample was also evaluated for plasma Rspon1 and Slit2 by an ELISA assay kit.

The patients received the following surgical procedures: twenty-two patients received a combination of radical hysterectomy $(\mathrm{RH})$, bilateral salpingo-oophorectomy (BSO) and pelvic lymph node dissection; eleven patients underwent RH, and pelvic lymph node dissection; and one patient received modified-RH, BSO, and pelvic lymph node dissection.

Immediately following surgery, information regarding the mode of surgery (laparoscopy, or laparotomy), amount of blood loss during surgery, operation time (in minutes), FIGO stage, cell type, histologic grade, tumor size, LVSI was recorded. Tissue samples were embedded in paraffin and kept for further analysis.

Two weeks after the start of chemotherapy or radiotherapy, another $10 \mathrm{~mL}$ of blood was collected for the plasma Rspond1 and Slit2 measurements, as well as for routine blood counts (including platelet counts, mean platelet volume, hematocrit, neutrophil count, aPTT, PT, TT, fibrinogen, INR, D-dimer (if tested), WBC, and HB levels). In addition, the RTOG acute radiation morbidity on different tissues/organs, such as skin, mucous membrane, eye, ear, salivary gland, pharynx and esophagus, larynx, upper and lower GIs including pelvis, lung, genitourinary, heart, and CNS, were evaluated and scored.

At the conclusion of the postoperative chemotherapy and/or radiotherapy treatment, the name, dose and duration of chemotherapy was recorded, and the dose, schedule, and overall duration of the external beam radiotherapy (EBRT) as well as high-dose rate (HDR) intracavitary brachytherapy
(ICBRT) and total effect for the dose schedule was recorded. A final blood sample was drawn for the plasma Rspo1 and Slit2 measurements and for further routine blood counts (including platelet counts, mean platelet volume, hematocrit, neutrophil count, aPTT, PT, TT, fibrinogen, INR, D-dimer (if tested), WBC, and HB levels). The RTOG acute morbidity scores were evaluated again. After completion of treatment, the patients were followed up regularly with examinations at $6,12,24,36,48$, and 60 months after surgery.

\section{ELISA}

The blood samples were centrifuged by $2,000 \mathrm{~g}$ for 10 minutes and the supernatant was collected. Slit 2 and Rspo1 serum expression levels were measured by ELISA kits (Cloud-Clone Corp., Wuhan, Hubei, China; R\&D Systems, Inc., Minneapolis, MN, USA, respectively) according to the manufacturer's instructions (19). The amount of these cytokines was quantified as the absorption at $450 \mathrm{~nm}$. All samples were evaluated in duplicate.

\section{Statistical analysis}

For the descriptive statistics, a boxplot was used to graphically depict the Rspo1 and Slit2 expression data groups, in which the bottom and top of the box represent the lower and upper quartiles, respectively, the band near the middle of the box represents the median, and the ends of the whiskers represent the smallest and the largest nonoutlier observations (20). To evaluate the effect of Rspo1 and Slit2 expression levels and other possible factors related to radiosensitivity, a logistic regression model was used. $\mathrm{P}$ values $<0.05$ were considered statistically significant. All computations were made with $\mathrm{R}$ 4.0.4.

\section{Results}

\section{Patient characteristics}

Patient baseline characteristics, including age at diagnosis, body mass index (weight/height), FIGO stage, type of operation, operation time, and estimated blood loss are listed in Table 1. The age of patients ranged from 31 to 57 years old $(44.9 \pm 6.1)$ years old. The median body mass index was 22.7 . There were $61.8 \%, 11.8 \%, 23.5 \%$, and $2.9 \%$ patients in FIGO IB1, IB2, IIA1, and IIA2 stages, respectively. Post-surgery, 27 patients received alternating 


\section{Page 4 of 9}

chemotherapy and radiotherapy, three patients received chemotherapy alone, and four patients were treated with radiotherapy alone. All the 27 patients who received combined chemotherapy and radiotherapy received chemotherapy for the first round, radiotherapy, and then chemotherapy for the second round. The time interval between adjacent therapies was 3 weeks.

Table 1 Patient characteristics

\begin{tabular}{lc}
\hline Characteristic & Patients \\
\hline Age (years) & $44.9 \pm 6.1[31-57]$ \\
Body mass index (weight/height/ & $22.7 \pm 2.5(17.8-28.3)$ \\
height) & \\
FIGO stage & $21(61.8 \%)$ \\
IB1 & $4(11.8 \%)$ \\
IB2 & $8(23.5 \%)$ \\
IIA1 & $1(2.9 \%)$ \\
IIA2 & $22(64.7 \%)$ \\
Type of operation & $11(32.4 \%)$ \\
RH + BSO + pelvic lymph node & $1(2.9 \%)$ \\
dissection & \\
RH + pelvic lymph node dissection & $173.4 \pm 44.2(100.0-274.0)$ \\
Modified-RH + BSO + pelvic lymph & $300.4 \pm 419.2(100-2,400)$ \\
node dissection & \\
Operating time (min) & \\
Estimated blood loss (mL) & \\
\hline
\end{tabular}

FIGO, International Federation of Gynecology and Obstetrics.
Nie et al. R-spondin 1 and Slit2 in cervical cancer adjuvant therapy

\section{Rspo1 and Slit2 serum levels}

The serum levels of Rspo1 and Slit2 following the first chemotherapy, radiotherapy, or second chemotherapy round were detected by ELISA assay. As shown in Figure 1A, the average concentrations of Slit2 were $8.15,7.97$, and $7.69 \mathrm{ng} / \mathrm{mL}$, indicating insignificant level changes between each chemotherapy or radiotherapy treatment. Likewise, Rspo1 levels were 105.81, 103.02, and $101.50 \mathrm{pg} / \mathrm{mL}$ after the first chemotherapy, radiotherapy, or second chemotherapy round, and did not show a statistically significant difference (Figure 1B).

\section{Morbidity scores and number of therapies}

The acute radiation morbidity scoring criteria were used to evaluate the efficiency of treatment after the first chemotherapy (CT1), radiotherapy (RT), or the second chemotherapy (CT2). As shown in Figure $2 A$, compared to a zero score at CT1, the neurological sensory score rose to one after RT or tCT2. However, there were no differences between RT and CT2. The mean infection scores after CT2 were significantly higher than CT1 and RT, but statistically there was no significant difference between CT1 and RT (Figure 2B).

\section{The correlation between Rspo1 and clinical pathological parameters}

The concentration of Rspo1 levels was detected using an ELISA assay afterCT1. The concentration of Rspo1
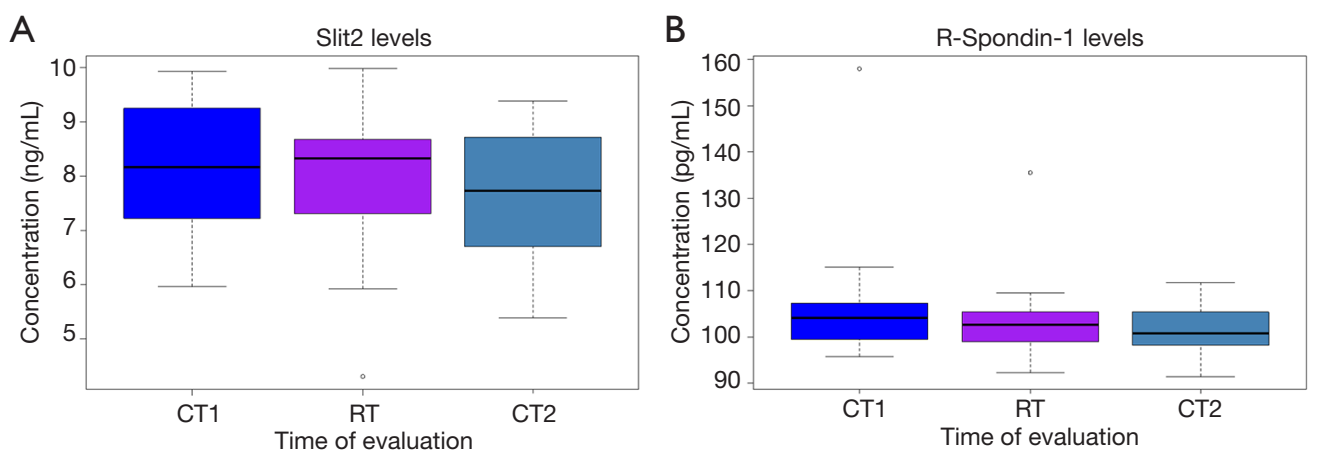

Figure 1 Rspo1 and Slit2 expression level in serum. Neither Slit2 levels (A) nor Rspo11 levels (B) changed significantly after CT1, radiotherapy, or CT2. 

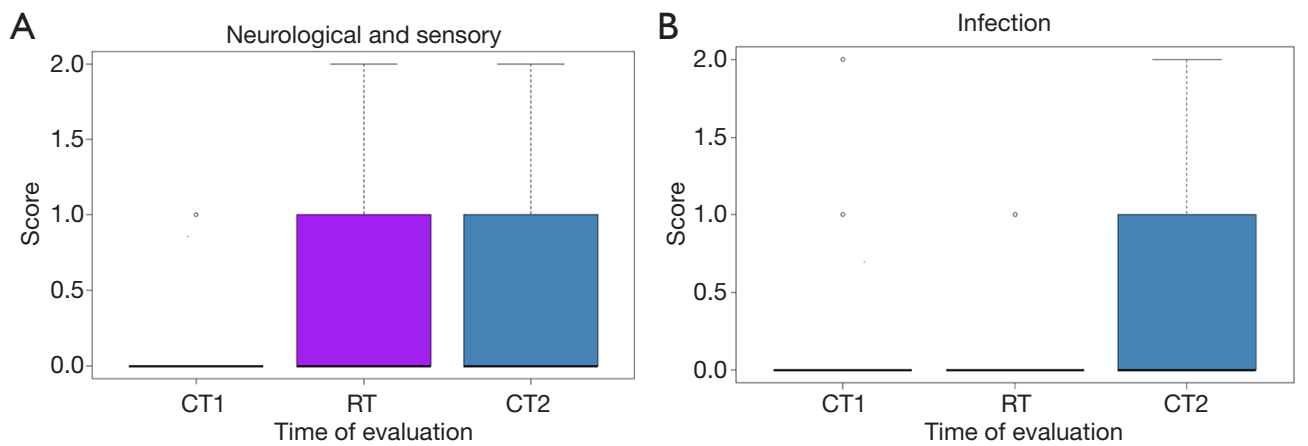

Figure 2 Morbidity scoring and number of therapies. Neurological sensory scores and influence of infection scores were elevated with the increasing number of therapies. The neurological sensory score rose from zero at CT1 to one after RT or CT2 (A), but there was no statistically significant difference between CT1 and RT (B).

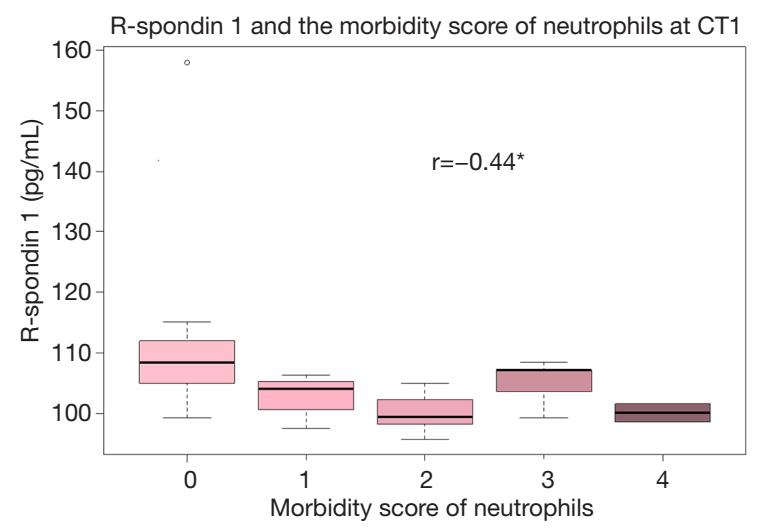

Figure 3 The levels of R-spondin 1 inversely correlates to the morbidity score of neutrophils at CT1. *, $\mathrm{P}<0.05$.

decreased while the morbidity score of neutrophils increased. The correlation coefficient between Rspo1 and the morbidity score of neutrophils at CT1 was -0.44 (Figure 3), which suggested that the level of Rspo1 was inversely correlated with the morbidity score of neutrophils at CT1.

As shown in Figure $4 A$, correlation coefficient between Rspo1 and the morbidity score of neutrophils at RT was -0.46 , which indicated the level of Rspo1 reversibly correlates to the morbidity score of neutrophils at RT. The mean (and SD) of the Rspo1 staining level was $104 \mathrm{pg} / \mathrm{mL}$, while the morbidity score of neutrophils was zero. When the morbidity score of neutrophils increased above two, the staining level of Rspo1 decreased to less than $100 \mathrm{pg} / \mathrm{mL}$. When comparing the Rspo1 level to the morbidity score of $\mathrm{HB}$ after RT, if the morbidity score of $\mathrm{HB}$ was zero, the Rspo1 concentration was $105.00 \mathrm{pg} / \mathrm{mL}$, and if the morbidity score of HB increased to one, the Rspo1 level was equal to zero. However, if the HB score increased to two (Figure 4B), the serum Rspo1 concentration decreased to less than $100 \mathrm{pg} / \mathrm{mL}$. In conclusion, the levels of Rspo1 were negatively correlated to the morbidity score of $\mathrm{HB}$. Meanwhile, the level of Rspo1 also reversibly correlated to the morbidity score of platelets (Figure 4C). The high morbidity score of platelets was achieved when Rspol level is low.

The correlation coefficient between Rspo1 and the clinical pathological parameters was also evaluated. As shown in Figure 4D,E, F, when the neurological/sensory score was greater than one, the Rspo1 staining level significantly decreased to less than $100 \mathrm{pg} / \mathrm{mL}$ after CT2. In summary, Rspo1 levels were inversely correlated with the morbidity score of neutrophils, the infection score $(r=-0.42)$, and the performance status at CT2.

\section{The correlation between Slit2 and clinical pathological parameters}

We also examined the relationship between Slit2 and the clinical pathological parameters. The correlation coefficient between Slit 2 and the cardiovascular score at RT was -0.42 (Figure $5 A$ ), the correlation coefficient between Slit2 and the reproductive/urinary score at RT was -0.50 (Figure $5 B$ ), and the correlation coefficient between Slit2 and the neurological/sensory score at RT was -0.58 (Figure 5C). These results indicated that the level of Slit2 correlated negatively with the heart, genitourinary, and neurological sensory scores following RT. Based on the correlation coefficient analysis, the Slit2 levels inversely correlated with the upper GI tract score at CT2 with $\mathrm{r}=-0.61$ 

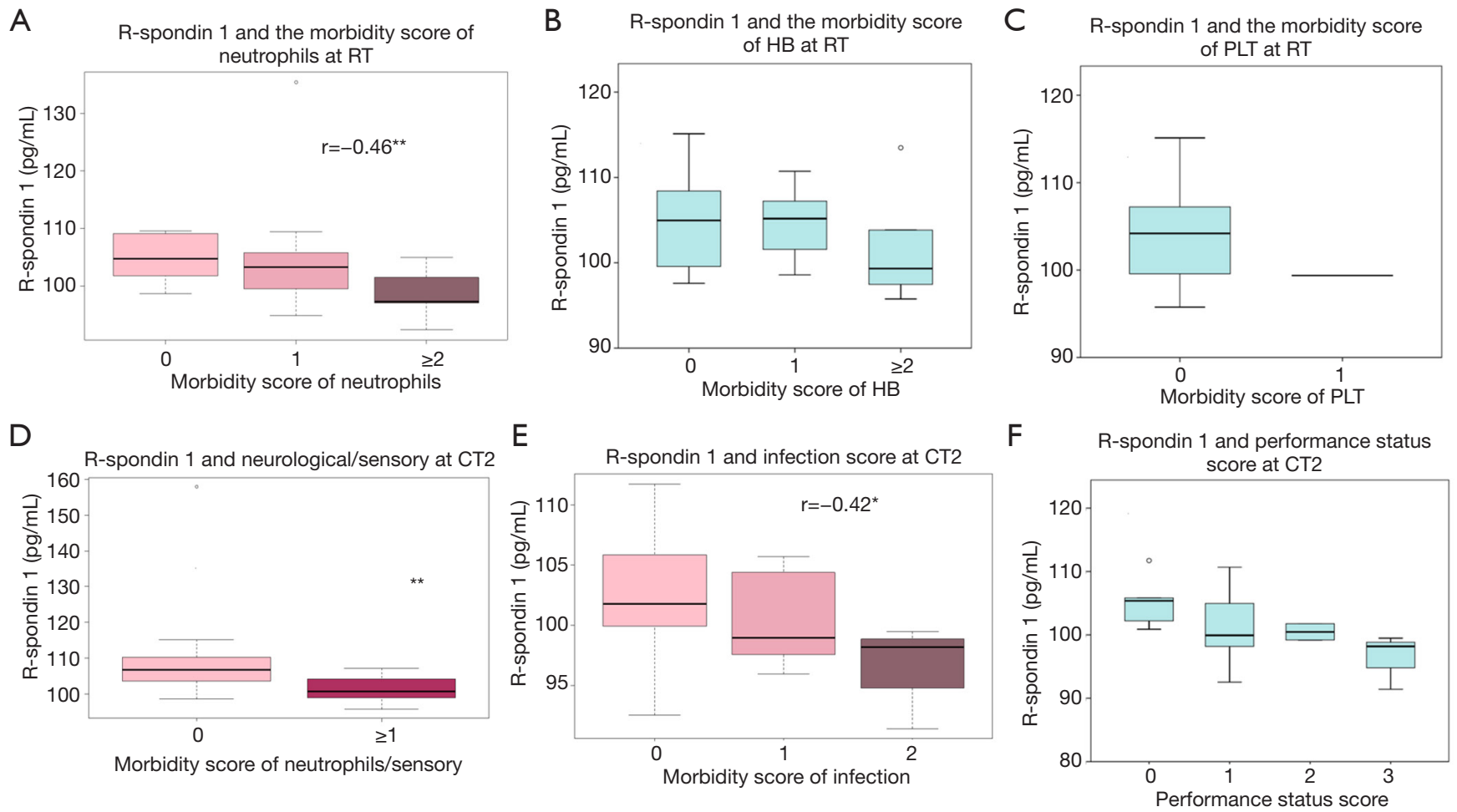

Figure 4 The correlation between Rspo1 and clinical pathological parameters. The levels of R-spondin 1 inversely correlates to the morbidity score of neutrophils (A), the morbidity score of hemoglobin (B), and the morbidity score of platelets (PLT) (C) following RT. The levels of Rspo1 inversely correlates to the neurological sensory score (D), infection score (E), and performance status (F) at $\mathrm{CT} 2 .{ }^{*}$, $\mathrm{P}<0.05$; **, $\mathrm{P}<0.01$.

(Figure 5D). Meanwhile, the heart and the morbidity score of HB decreased at CT2, which suggested the level of Slit2 was negatively correlated with the heart and the morbidity score of $\mathrm{HB}$ (Figure $5 E, F)$.

\section{Discussion}

Radiosensitivity varies among individual patients due to the heterogeneity of cervical cancer $(6,21,22)$. Numerous efforts have been made to identify predictive biomarkers and molecular targeted drugs although little progress has been made, especially in radiotherapy (7). Therefore, it is necessary to explore the biological mechanisms and related interactive factors associated with radiosensitivity (23). Recently, researchers have observed that Rsop1 and Slit2, which are promising indicators for the prediction of outcomes in advanced cervical cancer patients, have proven to play an important role in regulating the development, progress, and vasculogenesis of various tumors $(14,16,24)$. Therefore, this study aimed to explore the expression levels of Rspo1 and Slit2 and their correlation with clinicopathological features in postoperative cervical cancer patients.

We used ELISA kits to compare the serum Rspo1 and Slit2 levels of all patients and analyzed the correlation coefficient between the predicted biomarkers and the clinical pathological features. We found no significant changes of Rspo1 and Slit2 levels between CT1, RT or CT2 groups. However, the neurological sensory scores and influence of infection scores were elevated with increasing rounds of therapies. In addition, Rspo1 expression levels correlated negatively with the morbidity score of neutrophils, infection score, neurological sensory score and performance status at CT1 and CT2 separately. The Rspo1 level was also inversely correlated to the morbidity score of hemoglobin, platelet, and neutrophil following RT. Our results showed that human serum Slit2 was negatively correlated with the genitourinary, heart, and neurological sensory scores following RT, and inversely correlated to the upper GI tract score following CT2 with a statistically 
A

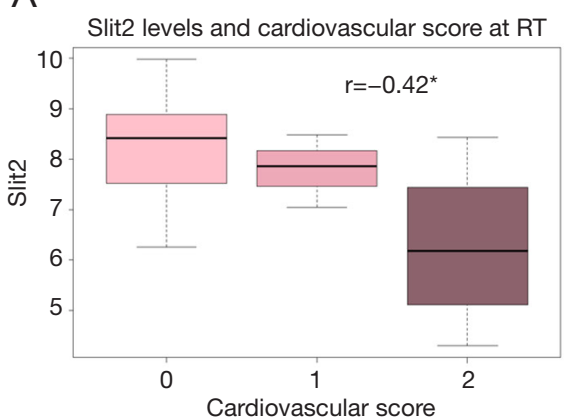

$\mathrm{D}$

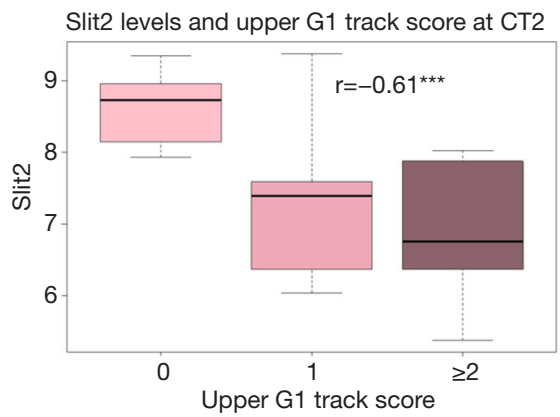

B

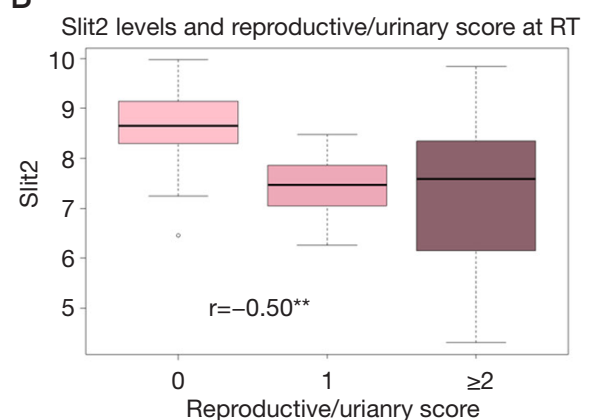

E

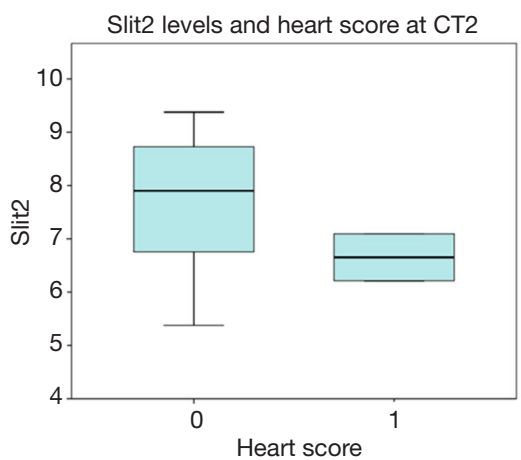

C

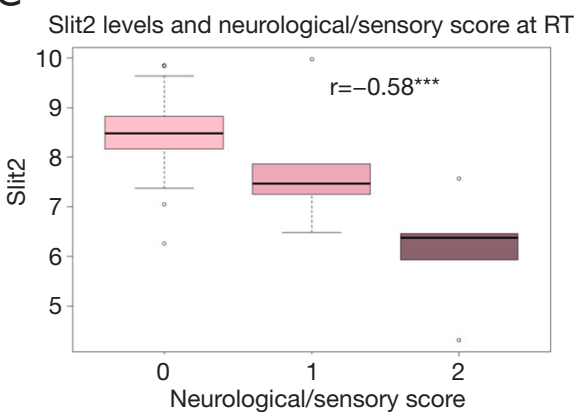

F Slit2 levels and the morbidity score of $\mathrm{HB}$ at CT2

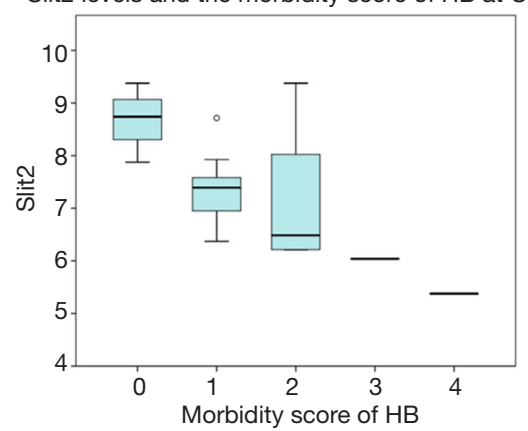

Figure 5 The correlation between Slit2 and clinical pathological parameters. The levels of SLIT2 correlate negatively with the heart (A), genitourinary (B), and neurological sensory scores (C) following RT. The level of SLIT2 inversely correlates with the upper G.I. score (D), heart (E), and hemoglobin level (F) at CT2. *, $\mathrm{P}<0.05 ;$ **, $\mathrm{P}<0.01$; ***, $\mathrm{P}<0.001$.

significant difference.

Slit2 was first characterized in neuronal development and was found to interact with Robol to mediate myogenesis, endothelial cell migration (25), and leukocyte chemotaxis (26). Slit2 has been shown to induce tumor angiogenesis and to regulate lymphangiogenesis in various human cancers $(11,27)$. A previous study reported that a short pulse of Rspo1 plus Slit2 reduced intestinal stem cell loss, mitigated gut impairment, and prolonged overall survival in mice without concomitantly decreasing tumor sensitivity to chemotherapy (14). The results of our study are consistent with previous studies on Rspo1 and Slit2, since they were negatively correlated with neutrophil and neurological sensory scores. From our results, we suggest Rspo1 plus Slit2 may have a specific role in monitoring the tolerance of cervical cancer patients to aggressive chemotherapy and/or radiotherapy.

This study clearly showed that the levels of Rspo1 and Slit2 correlate positively to the treatment tolerance of patients. Conversely, Rspo1 and Slit2 levels showed a negative correlation to the morbidity score of the patients undergoing chemotherapy and/or radiotherapy. We could not evaluate the exact roles and mechanisms of Slit2 and Rspo1, and therefore future work is warranted to delineate the secretory mechanisms of Rspo1 and Slit2 and their function in cell apoptosis. In conclusion, these preliminary results suggest Rspo1 and Slit2 may be potential predictive biomarkers for cervical cancer patients receiving chemotherapy or radiotherapy postoperatively, and our findings validate the pursuit of the clinical significance of Rspo1 and Slit2.

\section{Acknowledgments}

This project was initiated at the suggestion of Professor Jianguo Geng of the University of Michigan at Ann Arbor, whose work on R-spondin-augmented chemoradioprotection inspired us. We thank Professor Geng for his generosity in sharing his thoughts and insights. Funding: This research was supported by grant 2018YQ60 


\section{Page 8 of 9}

(JN) from the Shanghai Health System Talent Training Program.

\section{Footnote}

Reporting Checklist: The authors have completed the MDAR reporting checklist. Available at http://dx.doi.org/10.21037/ atm-21-87

Data Sharing Statement: Available at http://dx.doi. org/10.21037/atm-21-87

Conflicts of Interest: All authors have completed the ICMJE uniform disclosure form (available at http://dx.doi. org/10.21037/atm-21-87). The authors have no conflicts of interest to declare.

Ethical Statement: The authors are accountable for all aspects of the work in ensuring that questions related to the accuracy or integrity of any part of the work are appropriately investigated and resolved. All procedures performed in this study involving human participants were in accordance with the Declaration of Helsinki (as revised in 2013). The study was approved by the ethics board of Shanghai Obstetrics and Gynecology Hospital of Fudan University (No.: 2021-27) and informed consent was taken from all the patients.

Open Access Statement: This is an Open Access article distributed in accordance with the Creative Commons Attribution-NonCommercial-NoDerivs 4.0 International License (CC BY-NC-ND 4.0), which permits the noncommercial replication and distribution of the article with the strict proviso that no changes or edits are made and the original work is properly cited (including links to both the formal publication through the relevant DOI and the license). See: https://creativecommons.org/licenses/by-nc-nd/4.0/.

\section{References}

1. Baeyens A, Thierens H, Claes K, et al. Chromosomal radiosensitivity in breast cancer patients with a known or putative genetic predisposition. Br J Cancer 2002;87:1379-85.

2. Torre LA, Bray F, Siegel RL, et al. Global cancer statistics, 2012. CA Cancer J Clin 2015;65:87-108.

3. Burd EM. Human papillomavirus and cervical cancer. Clin Microbiol Rev 2003;16:1-17.
Nie et al. R-spondin 1 and Slit2 in cervical cancer adjuvant therapy

4. Eifel PJ, Winter K, Morris M, et al. Pelvic irradiation with concurrent chemotherapy versus pelvic and paraaortic irradiation for high-risk cervical cancer: an update of radiation therapy oncology group trial (RTOG) 90-01. J Clin Oncol 2004;22:872-80.

5. Chemoradiotherapy for Cervical Cancer Meta-analysis Collaboration (CCCMAC). Reducing uncertainties about the effects of chemoradiotherapy for cervical cancer: individual patient data meta-analysis. Cochrane Database Syst Rev 2010;2010:CD008285.

6. Luo YM, Xia N, Yang L, et al. CTC1 increases the radioresistance of human melanoma cells by inhibiting telomere shortening and apoptosis. Int J Mol Med 2014;33:1484-90.

7. Yim EK, Park JS. Biomarkers in cervical cancer. Biomarker Insights 2007;1:215-25.

8. Brose K, Bland KS, Wang KH, et al. Slit proteins bind Robo receptors and have an evolutionarily conserved role in repulsive axon guidance. Cell 1999;96:795-806.

9. Wang KH, Brose K, Arnott D, et al. Biochemical purification of a mammalian slit protein as a positive regulator of sensory axon elongation and branching. Cell 1999;96:771-84.

10. Wu W, Wong K, Chen JH, et al. Directional guidance of neuronal migration in the olfactory system by the protein Slit. Nature 1999;400:331-6.

11. Wang B, Xiao Y, Ding BB, et al. Induction of tumor angiogenesis by Slit-Robo signaling and inhibition of cancer growth by blocking Robo activity. Cancer Cell 2003;4:19-29.

12. Bedell VM, Yeo SY, Park KW, et al. roundabout4 is essential for angiogenesis in vivo. Proc Natl Acad Sci U S A 2005;102:6373-8.

13. Wang LJ, Zhao Y, Han B, et al. Targeting Slit-Roundabout signaling inhibits tumor angiogenesis in chemicalinduced squamous cell carcinogenesis. Cancer Science 2008;99:510-7.

14. Zhou WJ, Geng ZH, Spence JR, et al. Induction of intestinal stem cells by R-spondin 1 and Slit2 augments chemoradioprotection. Nature 2013;501:107-11.

15. Zhao J, De Vera J, Narushima S, et al. R-spondin1, a novel intestinotrophic mitogen, ameliorates experimental colitis in mice. Gastroenterology 2007;132:1331-43.

16. Zhao J, Kim KA, De Vera J, et al. R-Spondin1 protects mice from chemotherapy or radiation-induced oral mucositis through the canonical $\mathrm{Wnt} / \beta$-catenin pathway. Proc Natl Acad Sci U S A 2009;106:2331-6.

17. Bhanja P, Saha S, Kabarriti R, et al. Protective role of 
R-spondin1, an intestinal stem cell growth factor, against radiation-induced gastrointestinal syndrome in mice. PLoS One 2009;4:e8014.

18. Kim KA, Kakitani M, Zhao J, et al. Mitogenic influence of human R-spondin 1 on the intestinal epithelium. Science 2005;309:1256-9.

19. Kang YE, Choung S, Lee JH, et al. The Role of Circulating Slit2, the One of the Newly Batokines, in Human Diabetes Mellitus. Endocrinol Metab (Seoul) 2017;32:383-8.

20. Buttarazzi D, Pandolfo G, Porzio GC. A boxplot for circular data. Biometrics 2018;74:1492-501.

21. Meng XY, Liao Y, Liu XP, et al. Concurrent cisplatinbased chemoradiotherapy versus exclusive radiotherapy in high-risk cervical cancer: a meta-analysis. Onco Targets Ther 2016;9:1875-88.

22. Rantanen V, Grénman S, Kulmala J, et al. Characterization and radiosensitivity of UT-EC-2A and UT-EC-2B, two new highly radiosensitive endometrial cancer cell lines derived from a primary and metastatic tumor of the same

Cite this article as: Nie J, Shao J, Guo SW, Liu X. The relevance of plasma R-spondin 1 and Slit2 as predictive biomarkers in cervical cancer chemotherapy and radiotherapy. Ann Transl Med 2021;9(10):837. doi: 10.21037/atm-21-87 patient. Gynecol Oncol 1995;56:53-62.

23. Noordhuis MG, Eijsink JJ, Roossink F, et al. Prognostic cell biological markers in cervical cancer patients primarily treated with (chemo) radiation: a systematic review. Int J Radiat Oncol Biol Phys 2011;79:325-34.

24. Ootani A, Li X, Sangiorgi E, et al. Sustained in vitro intestinal epithelial culture within a Wnt-dependent stem cell niche. Nat Med 2009;15:701-6.

25. Kramer SG, Kidd T, Simpson JH, et al. Switching repulsion to attraction: changing responses to slit during transition in mesoderm migration. Science 2001;292:737-40.

26. Wu JY, Feng L, Park HT, et al. The neuronal repellent Slit inhibits leukocyte chemotaxis induced by chemotactic factors. Nature 2001;410:948-52.

27. Yang XM, Han HX, Sui F, et al. Slit-Robo signaling mediates lymphangiogenesis and promotes tumor lymphatic metastasis. Biochem Biophys Res Commun 2010;396:571-7.

(English Language Editor: D. Fitzgerald) 